\title{
What does a Culture of Care look like? Lessons learnt from a workshop survey
}

\author{
There is a growing interest in being able to better describe a Culture of Care, particularly as institutions are being \\ asked to nurture such a culture and engage proactively on the care and welfare of the animals used for research \\ purposes. Based on responses collected using an anonymized virtual interactive online poll, we provide some \\ suggestions to help promote further awareness of how animal welfare ethical review bodies can champion a \\ Culture of Care.
}

\section{Sally Robinson and Angela Kerton}

n the context of animal research, the term 'Culture of Care' is used to describe an establishment-wide commitment to improving animal welfare, scientific quality, care of the staff, and transparency for all stakeholders, including the public. . $^{1,2}$

A good Culture of Care is recognized as being key to the welfare of the animals in research facilities and central to the recognition and support of staff working both directly and indirectly with the animals. A good Culture of Care can also improve the quality of the scientific data obtained from the animals used.,4 Institutional support is critical to provide a safe, and supportive framework to promote a caring ethos.

In Europe, the Animal Welfare Body (AWB) and in the UK, the Animal Welfare and Ethical Review Body (AWERB) should have an active role in facilitating the exchange of perspectives and requiring that the professional standards of care and staff development are the same for everyone. The current EU Directive guidance on AWB reinforces their role in promotion and maintenance of a Culture of Care; this includes the need for shared responsibility, the need for a pro-active approach towards improving standards rather than merely reacting to problems when they arise, and the importance of effective communication throughout the establishment. ${ }^{5}$ These attributes are largely behavioral. Having measurable indicators of good practice can help build a Culture of Care in a more systematic fashion., ${ }^{2,6}$

Using anonymized examples collected during a 2021 workshop, our aim is to discuss some practical actions to implement and assess an institution's Culture of Care and to reference useful frameworks that provide good practice indicators. Whilst our workshop had a UK focus, we believe that a number of the roles an AWERB can play in promoting and maintaining a Culture of Care can broadly be applied across other oversight bodies, including Institutional Animal Care and Use Committees (IACUC) in the United States.

\section{Results and reflections from the workshop}

During February 2021, over 90 delegates attending a workshop on the topic of Culture of Care in the UK were invited to anonymously respond to a series of questions (See Supplemental Information). The attendees were largely UK-based academic and industrial (e.g. pharmaceutical company) animal care staff, lab managers, researchers, AWERB members, veterinarians and regulatory compliance staff. We highlight the responses to some of the questions below along with our reflections and suggestions for Animal Welfare and Ethical Review Bodies (AWB in Europe).

Question \#1: My Establishment has a written policy / statement on animal welfare that specifically refers to a Culture of Care. Just over half of the respondents answered yes to this question. Many organizations have written policies on animal welfare that may align with organizational values, such as doing the right thing. ${ }^{7,8}$ The AWERB may be responsible for producing or contributing to such policies. For a Culture of Care to be nurtured, it is essential that such policies define this concept and emphasize its importance for animals, staff and science. Such policies can and should set the tone for expected values and behaviors within an establishment. The policy should be reviewed and updated to keep it as a living reflection of the values and behaviors of the organization.

Question \#2: It is possible to benchmark a Culture of Care? Nearly all respondents thought it might be possible to benchmark a Culture of Care. We would like to highlight that there are objective indicators of a Culture of Care that can be reviewed, assessed and used to drive improvements in establishments. ${ }^{2,6}$ Readers are strongly encouraged to familiarize themselves with these indicators. A list of indicators that relate to the AWB itself can be found in Robinson et al.; ${ }^{2}$ selected examples can be found in Table 1.

Additionally, a leaflet produced by the European Federation of the Pharmaceutical Industry and Associations is also available, which provides some tips on how to get started discussing and assessing your Culture of Care. ${ }^{9}$

Question \#3: My Establishment has a policy for raising any concerns which is visible to staff (e.g. on prominent display in the animal facility) and easy to follow. Approximately $75 \%$ of the responses agreed or strongly agreed with the statement. A positive Culture of Care involves all staff being able to speak up, being listened to and having any questions or concerns responded to. Staff should be able to raise concerns openly and without blame in the spirit of learning and continuous improvement. Routes to accomplish this can include having direct named contacts to speak to, such as a member of the AWERB or managers, as well as local and/or organizational mechanisms to raise concerns; these should be independent and confidential. Confidential and independent means of raising concerns provide 'psychological safety', even if these options are never used. The chosen mechanism(s) should be reviewed regularly to keep them up to date, and always made visible and accessible to staff. The AWERB has a role here to ensure such mechanisms are in place and that they work effectively.

Question \#4: The culture in my organization allows me to challenge researchers about animal welfare. The response to this question was positive with $90 \%$ of responses agreeing or strongly agreeing with the statement. 


\section{Table 1 | Examples of objective indicators for assessing an institution's Culture of Care}

AWB visibility within the establishment

AWB resource

AWB membership

AWB annual goals

AWB Transparency
Demonstrate a commitment to Culture of Care, for example, by visiting the animal facility, talking to staff, hosting local events and having an AWB poster on display so all staff know who to contact.

This might include a budget to support continuing education of AWB members, to invite external speakers or fund continued professional development (CPD) sessions and provide recognition awards.

A diverse membership is encouraged with lay and independent members to enable different ethical perspectives to be considered. It is strongly recommended that animal care staff are members of the AWB as this enables their voices to be heard. An independent chair can provide neutrality in the meeting discussions.

Setting annual goals, such as sponsoring a subgroup to consider a Culture of Care, demonstrates a proactive approach to improving standards.

Meetings are open. Dates of meetings, agendas and minutes are accessible to staff at the establishment. Allowing a wide number of staff to attend supports a forum for discussing ethical, scientific and welfare topics
It is important that those who are responsible for both caring for animals and conducting procedures on animals have the opportunity to easily raise any challenges - or more often, simply ask questions for clarification and understanding. Questions and challenges should be addressed through discussions and sharing perspectives on the objectives of the study and expectations around management of welfare. A pre-study meeting is one way for these discussions to happen in a structured way. In addition, engaging animal care and technical staff on the outcomes of the study through a structured post-study feedback meeting can enhance engagement and job satisfaction.

Such interactions should be two-way, and researchers should equally be able to challenge the staff working with the animals about welfare or any other topics connected to the quality and integrity of the study. Two-way communication between researchers and those conducting the animal studies is essential, as sharing outcomes, any learning, and celebrating successes together is an important feature of a positive Culture of Care. The AWERB can have a role here by encouraging or requiring joint presentations and inputs of all perspectives into discussions.

\section{Question \#5: Our Animal Welfare Ethical} Review Body (AWERB) promotes a Culture of Care. Approximately 80\% of responses answered yes to this question; which on first sight was extremely positive. In a follow up question, we delved deeper and asked for examples of how the AWERB promotes a Culture of Care.

A number of attendees cited activities that might be considered a baseline for compliance - e.g., having a Named
Veterinary Surgeon and Named Animal Care and Welfare Officer report at the AWERB meeting, or following up on non-compliance events. However, a Culture of Care goes beyond maintaining compliance.

Other respondents cited 3Rs activities, including poster events, talks, and awards. A strong drive for improvement in the 3Rs is one indicator of a positive Culture of Care. However, this is often conflated with a Culture of Care and the two things are different. A Culture of Care more broadly describes the culture of the establishment that supports staff, animal welfare, open communications, transparency and high quality science. Having a strong Culture of Care provides the framework that will enable the behaviors that drive continuous improvement in the application of the 3Rs.

Other responses highlighted some good ideas that have the potential to directly promote a Culture of Care that have been implemented elsewhere, such as, creating a Culture of Care focus group sponsored and overseen by the AWERB, having AWERB annual goals to improve Culture of Care, holding open AWERB meetings allowing for broader inclusion of staff views, developing a Culture of Care pledge activity sponsored by the AWERB, and identifying an AWERB Culture of Care Champion.

A minority of responses indicated that their AWERB did not promote a Culture of Care and provided several reasons, which included that a Culture of Care was never discussed on their meeting agendas, it was not considered a priority amongst other tasks, and that there was limited understanding of what the term means.

Question \#6: Our AWERB has clear communication methods that help support a Culture of Care, e.g. local newsletter, publication of minutes or internal website. The responses to this question was variable across the scale. Approximately $40 \%$ of responses agreed or strongly agreed whilst just under $20 \%$ disagreed or strongly disagreed. One way that establishments can support a Culture of Care is by developing communication methods, such as a local newsletter or internal website. Regular communications from the AWERB should be shared e.g. AWERB goals and meeting updates and newsletters that include for example staff recognition, development opportunities, well-being support and dissemination of 3 Rs activities and progress.

Question \#7: My organization provides staff with opportunities for professional development e.g. to find good practice in literature, attend conferences, through exchange visits. The response to this question was variable with just $67 \%$ of the responses in the 'agree' and 'strongly' agree category. All staff should be supported in their professional development including those who care for animals. They should be members of appropriate professional societies (e.g. animal technology societies) and have individual development plans. Recognizing that animal care and technical staff have a competence and expertise that is a prerequisite for good science provides a rationale for funding individual subscriptions and individual development planning, in the same way scientists are expected to be members of scientific societies and have individual development plans. ${ }^{2}$ In some organizations there is less funding and support for animal care and technical staff development than there is for scientists. The AWERB can play a role here by advising the Establishment Licence Holder (Institutional Official in USA) about the budgets and resources required to adequately support staff training and development.

\section{Question \#8: My organization actively} promotes the $3 \mathrm{Rs}$ in working practices. $60 \%$ of responses agreed or strongly agreed with this statement.

Question \#9: My establishment formally celebrates the $3 R$ s and care excellence. The responses were equally split across yes and no to this statement.

Having the culture that supports continuous improvement in the application of the 3Rs is one indicator of a Culture of Care. In the UK the application of the $3 R$ s is also a requirement in animal research. The responses to the questions 8 and 9 indicate some room for improvement. This 
is an area where the AWERB can play an active role at the establishment-wide level to help drive specific initiatives, disseminate 3Rs information and promote the widest possible uptake. ${ }^{10}$ The UK National Centre for Replacement, Refinement and Reduction of Animals in Research (NC3Rs) has developed two self-assessment tools, one of which is aimed at the institutional level and would be an invaluable resource for further improving the uptake of new 3Rs approaches. ${ }^{11}$

Running or sponsoring a 3Rs award scheme is one way that AWERBs can support staff to put 3Rs into practice. An award scheme isn't just about the winners; it's effectiveness in supporting putting the 3 Rs into practice relies on a) a demonstration of commitment to the 3Rs from senior leaders and researchers and $b$ ) sharing all the entries and communicating broadly about the work, e.g. through poster roadshows and presentations. ${ }^{12}$

Question \#10: How important is staff well-being at your organization? The responses to this question were relatively positive with $50 \%$ stating this is the most important thing. There has been a strong emphasis on staff well-being through the Covid-19 pandemic. However, there are very few specific resources that are directed at the emotional burdens that can come when using animals in research.

One specific resource that is being developed is led by the Animal Research Nexus Programme. ${ }^{13}$ Care-full stories' is at a pilot stage. The aim of the Care-full stories project is to develop and pilot new training resources to help those working in the animal research sector to more fully and reflexively recognize, participate in and promote a culture of care in their working environment. The project uses fictionalized storytelling to facilitate participants to share their own stories of working in animal research and through this process build connections and a shared Culture of Care across different communities within the animal research facility.$^{14,15}$

The AWERB can have a role to ensure that appropriate support and resources are available and that staff are aware of these and can encourage staff to discuss their feelings about the research they are involved in without judgement. The AWERB can also ensure there are recognition systems in place that promote proactive and positive behaviors and recognize care at the cage-side and towards colleagues in addition to scientific achievements.

\section{Discussion and Conclusion}

The local AWERB underpins the tone, governance, systems and processes of an animal research program. When working well, this structure provides tangible support so that those who work directly and indirectly with animals can actively help each other. However, using a framework of structured good practice indicators can help organizations assess their Culture of Care, identify gaps and strive for improvement. These good practice indicators can help bring some structure to building a Culture of Care and would be a good basis to start discussions within your establishment. ${ }^{2,6}$

We reiterate the importance for providing better opportunities for open and safe discussions on feelings and emotions among the professionals working in the field of laboratory animal research. More attention should be given for recognizing and rewarding positive behaviors and actions of staff. We must continue raising awareness and developing resources to provide appropriate and sufficient care and support for the staff working with laboratory animals, as this impacts on better general wellbeing, for animals and staff.

We conclude that a larger survey of laboratory animal science workplace culture would be valuable in order to understand perspectives more broadly including internationally. For example; workshops and anonymous interactive surveys could be conducted at international laboratory animal conferences. A positive Culture of Care can lead to committed and engaged staff that deliver high quality animal work. An organization's commitment to staff is shown in meaningful ways such as investment in professional development, and through recognition of behaviors that exemplify a Culture of Care. ${ }^{16}$ A Culture of Care motivates individuals to be their best professional selves, which in turn delivers better results.

\section{Sally Robinson ${ }^{1 凶}$ and Angela Kerton (D) 2 \\ ${ }^{1}$ Animal Sciences and Technologies, \\ Clinical Pharmacology and Safety Sciences, BioPharmaceuticals R\&D, AstraZeneca, Alderley Park, UK. ${ }^{2}$ The Learning Curve (Development) Ltd., P.O Box 140, Ware, Hertfordshire SG9 0ZN, UK.

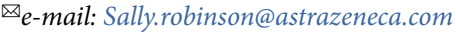

Published online: 30 August 2021

https://doi.org/10.1038/s41684-021-00852-6

References

1. Norecopa. Culture of Care. Available online: https://norecopa. no/more-resources/culture-of-care (accessed on 10 July 2021)

2. Robinson, S. et al. Lab Anim 54, 421-432 (2019). 2.

3. Boden, T. \& Hawkins, P. Animals Technology and Welfare $\mathbf{1 5}$ 151-156 (2016).

4. P, Hawkins. Communication and the Culture of Care: a sheet produced by the Culture of Care Network to facilitate better communication between scientists and animal technologists. Conference: Culture of Care Network. (2018). https://doi. org/10.13140/RG.2.2.33865.80481

5. National Competent Authorities for the implementation of Directive 2010/63/EU on the protection of animals used for scientific purposes. A working document on Animal Welfare Bodies and National Committees to fulfil the requirements under the Directive. http://ec.europa.eu/environment/ chemicals/lab_animals/pdf/endorsed_awb-nc.pdf (2014) accessed 28 July 2021.

6. Hawkins, P. \& Bertelsen, T. Animals 9, 969, https://doi.org/ 10.3390/ani9110969 (2019)

7. University of Leicester. Research Involving Animals. Division of Biomedical Services. Culture of Care. https://le.ac.uk/dbs/ animal-welfare/culture-of-care accessed 13 August 2021

8. Sanofi. Animal Protection Factsheet. https://www.sanofi.com/-/ media/Project/One-Sanofi-Web/Websites/Global/Sanofi-COM/ Home/common/docs/our-responsibility/documents-center/ factsheets-2020/animal-protection.pdf?la=en accessed 13 August 2021.

9. EFPIA. Living Your Culture of Care. https://www.efpia.eu/ media/602714/culture-of-care-2021-leaflet.pdf a13. accessed 27 July 2021

10. Robinson, S. Single Use of Needles: How AWERBs Can Support Refinements in Practice. https://www.nc3rs.org.uk/news/ single-use-needles-how-awerbs-can-support-refinements practice accessed 28 July 2021

11. UK National Centre for Replacement, Reduction and Refinement in Animal Research 3Rs Self-Assessment Tools. https://3rsselfassessment.nc3rs.org.uk/. Accessed 28 July 2021

12. Finnemore, P., Owen, S. Using an award scheme to promote 3 Rs innovation. https://nc3rs.org.uk/news/using-award-schemepromote-3rs-innovation Accessed 28 July 2021

13. The Animal Research Nexus. https://animalresearchnexus.org/ Accessed 10 August 2021

14. Greenough, B., Mazhary, H. Care-full Stories: Innovating a new resource for teaching a culture of care in animal research facilities. https://animalresearchnexus.org/publications/care-fullstories-innovating-new-resource-teaching-culture-care-animalresearch Accessed 29 July 2021

15. Greenhough, B. Telling care-full stories? Exploring how we can use the insights of our work to help build and sustain cultures of care in animal research. https://sso.services.box.net/sp/ ACS.saml2 Accessed 29 July 2021

16. Booth Cole, M. A Culture of Care, Without Compromise Stanford Social Innovation Review (2015) Available at: https:// ssir.org/articles/entry/a_culture_of_care_without_compromise Accessed July 2021

Acknowledgements

The authors acknowledge LASA and its participants for the interactive workshop session.

\section{Competing interests}

The author(s) declared no potential conflicts of interest with respect to the research, authorship, and/or publication of this report.

\section{Additional information}

Supplementary information The online version contains supplementary material available at https://doi.org/ 10.1038/s41684-021-00852-6. 